\title{
Inverse Kinematics for 6-DOF Manipulator by the Method of Sequential Retrieval
}

\author{
Liqing Nie \\ School of Mechanical and Power Engineering, Nanjing \\ University of Technology \\ Department of Vehicle Engineering, Nanjing Institute of \\ Technology, Nanjing, China \\ qingnlq@163.com
}

\author{
Qingjiu Huang \\ Institute of Robotic Science and Engineering, Nanjing \\ University of Technology, Nanjing, China \\ Department of Electrical Engineering, Kogakuin \\ University, Tokyo, Japan \\ huangqj@njut.edu.cn
}

\begin{abstract}
Analitical solution of the inverse kinematics has been found only for some particular structures of 6-DOF serial manipulator. We propose the Method of Sequential Retrieval by modifying the Effective Solution for Inverse Kinematics to solve the inverse kinematics for a 6-DOF robot arm which has not been analytically solved yet. We confirmed that our method can find some solutions which can not be solved by the Effective Solution for Inverse Kinematics. Moreover, we presented the effectiveness of our method by numerical analysis.
\end{abstract}

Key Words-Inverse Kinematics;6-DOF Manipulator;Method of Sequential Retrieval; Numerical Solution;ModificationWeight

\section{INTRODUCTION}

At present, robots play an active role in every field, but industrial use, use in hazardous environments, and use for collaboration in human society are their three major application fields.

In industrial applications, robots automatically perform jobs such as carrying, processing, and factory assembly, thereby contributing to promotion of efficiency in production.

Robots used in hazardous environments function in environments that are dangerous for humans such as rescue efforts at disaster sites, deep-sea investigation, space activities, and work around nuclear reactors. Human society collaboration robots are functioning actively in mascot and entertainment fields.

Industrial use robots and hazardous environment robots are designed and manufactured to serve specific functions. A robot manipulator is indispensable to attain their tasks. Regarding robot manipulators, arm-type manipulators are considered important elements in robot systems because of their wider movement range and higher general versatility.

Robot manipulator designs are of two categories: general purpose and exclusive work. For general-purpose robot manipulators, those with more than 6 degrees of freedom (DOF) are necessary to allow free positioning and posturing of hand tips (end effectors) to cope with jobs of every type. Although those with more than 7 DOF can handle tasks that are more complicated, their structures become increasingly more complicated with the difficulty of the task. For this reason, most general-purpose manipulators have 6 DOF. Those with less than 5 DOF are exclusive-use manipulators that are designed specifically with structures for tasks that can be performed even if restrictions are imposed on the posture and working range.

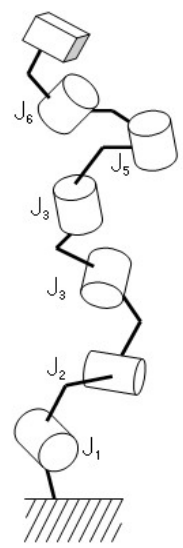

(a) Arbitrary structure

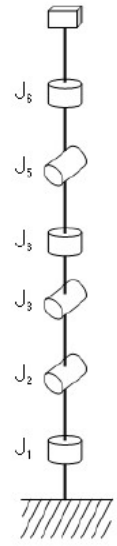

(b) PUMA

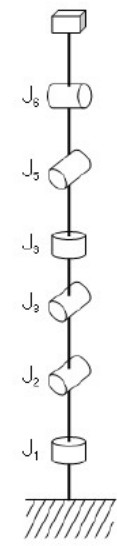

(c) RPY

type
Fig.1 Types of 6-DOF robot arm

The subject of this study is 6 DOF general-purpose robot arms, which we are investigating specifically. Fig.1 (a) shows that the general structure of a 6 DOF arm allows arbitrary length of each link and direction of joints. To move such a robot arm to do tasks of some kinds, it is necessary to obtain, from target values of hand tips, the angles of each joint corresponding to them, i.e. to solve so-called inverse kinematics. However, no analytical solution of the inverse kinematics applicable to arbitrary structure is available at present. Therefore, with most that are currently used for industrial applications, no analytical solution of the inverse kinematics has been found yet. Inverse kinematics can be solved analytically if all adjoining joint axes are parallel or orthogonal. Of those, the structure designated as PUMA-type as shown in Fig.1 (b). It is used extensively at present.

As described, one condition for practical application is that inverse kinematics must be solved, which imposes restrictions on selectable structures. In this study, for an RPY-type arm (Fig.1(c) ) which is one structure of inverse kinematics that has not been solved yet, we propose the Method of Sequential Retrieval, for which the modification weight is added to an efficient solution of the inverse kinematics to obtain a solution. 


\section{Definitions OF SyMBOLS}

First, to assure the accuracy of description of the problem, this section provides definitions of symbols used for this study. The problem is then described using these symbols.

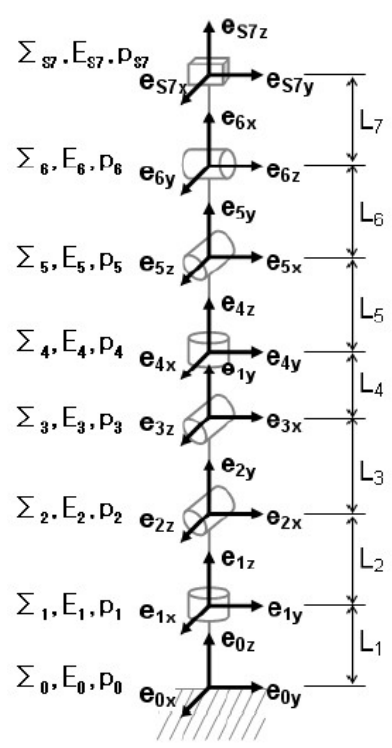

(a) Initial state(all angle of joints is $0[\mathrm{rad}])$

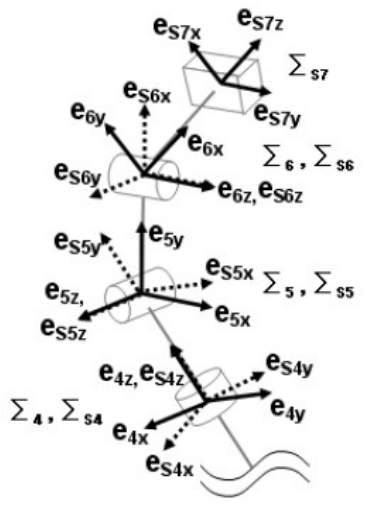

(b) $\Sigma_{i}$ (solid line) and

$\Sigma_{S i}($ dashed line) at certain angles
Fig.2 Definition of coordinate systems

Fig.2 (a) portrays a schematic diagram of an RPY-type arm when all joint angles are $0^{\circ}$. This diagram shows that root of the angle is designated by numeral 0 , each of the joints is represented by $1-6$, the hand tip by $\mathrm{S} 7$, each coordinate system by $\Sigma_{i}(\mathrm{i}=0, \ldots, \mathrm{S} 7)$, and the real world coordinate system by $\Sigma_{0}$. The position of the $\Sigma_{i}$ joint is represented $p_{i}$. The settings are made so that origin position of $\Sigma_{i}$ might agree with $p_{i}$. Furthermore, base vector of $\Sigma_{i}$ are respectively represented by $e_{i x}, e_{i y}$, and $e_{i z}$. A set of base vectors is represented by $E_{i}$. All coordinate systems are of Cartesian coordinates and are set so that $\mathrm{Z}$ direction $e_{i z}$ of the base vector agrees with the rotating axis. The length of each link (norm of $p_{i-} p_{i-1}$ ) is represented as $L_{i}$.

\section{A. Description of the problem}

Using symbols defined as described above, the inverse kinematics, which is the object of this paper, is described as shown below.

$$
\text { Given: }{ }^{0} p_{s 7},{ }^{0} E_{s 7},{ }^{i-1} E_{s i}, L_{i}(\mathrm{i}=1, \ldots, 7)
$$

Find: $\theta_{i}(\mathrm{i}=1, \ldots, 6)$

\section{Method of Sequential Retrieval}

Solving inverse kinematics of an RPY-type arm analytically is difficult. For such a structure, Newton-Raphson method and numerical solutions designated as efficient solution of the inverse kinematics (hereinafter designated as efficient solution) have been proposed. The efficient solution has features such as rapid convergence and that eight or fewer solutions are obtained. Additionally, it is considered that the efficient solution is characteristic in that solutions of a particular pattern can be selected. This feature plays an important role when the hand tip target positions and posture trajectories are converted to joint angle trajectories. However, a problem area of this efficient solution is that no convergence occurs depending on the target values. In this study, an improvement of the efficient solution, which is termed as the Method of Sequential Retrieval, is proposed.

\section{A. Outline of Method of Sequential Retrieval}

Using the Method of Sequential Retrieval, first, the initial value of each joint angle is given. Then two modes of processing are used, as described below.

Process 1: $\theta_{1}, \theta_{2}$ and $\theta_{3}$ are modified so that positions can become identical and

Process 2: $\theta_{4}, \theta_{5}$, and $\theta_{6}$ are modified so that postures can become identical.

They are repeated to achieve convergence of $\theta_{1}, \ldots, \theta_{6}$ values to meet with the target value. In an efficient solution, the positions and postures come to agree with the target value. However, in this study, the framework that is used is to set the modification amount using the modification weight $W p$, Wo. When $W p=1$ and $W o=1$, the same processing as that observed in the efficient solution is made. However, by setting an appropriate modification weight, it becomes possible to achieve convergence even for a case in which convergence cannot be made by the efficient solution. These operations are presented in Fig. 3 in flowchart form.

We adjust the hand tip position to the target value through modifications of $\theta_{1}, \theta_{2}$, and $\theta_{3}$. It is divided into two--Process 1.1 and Process 1.2-as depicted in Fig. 3. Although convergence judgment is provided between the two, its explanation is omitted here.

In the first half, Process 1.1, first, the position coordinates when $\theta_{1}, \ldots, \theta_{6}=\theta_{1}[k], \ldots, \theta_{6}[k]$ are calculated using direct kinematics $\Lambda_{6 \mathrm{p}}$. Direct kinematics are calculable using eq.(2) because the simultaneous conversion matrix ${ }^{0} A_{57}$ hand tip coordinates $\Sigma_{S 7}$ based on world coordinates $\Sigma_{0}$ can be expressed using a product of the conversion matrices of adjoining coordinates. Of those,position coordinates might take ${ }^{0} p_{S 7}$ in ${ }^{0} A_{S 7}$.

$$
\begin{aligned}
& { }^{0} A_{s 7}={ }^{0} A_{1}{ }^{1} A_{2}{ }^{2} A_{3}{ }^{3} A_{4}{ }^{4} A_{5}{ }^{5} A_{6}{ }^{6} A_{s 7} \\
& ={ }^{0} A_{s 1}{ }^{s 1} A_{1}{ }^{1} A_{s 2}{ }^{s 2} A_{2}{ }^{2} A_{s 3}{ }^{s 3} A_{3}{ }^{3} A_{s 3}{ }^{3} A_{s 4} \\
& { }^{s 4} A_{4}{ }^{4} A_{s 5}{ }^{s 5} A_{5}{ }^{5} A_{s 6}{ }^{s 6} A_{6}{ }^{6} A_{s 7}
\end{aligned}
$$

Next, difference $\Delta p_{S 7}$ with the target position is calculated. This is multiplied by weight $W p$ and is added to the current position ${ }^{0} p_{57}[k]$ to create a newly modified point ${ }^{0} p_{S 7}[k+1]$. Values of $\theta_{1}, \theta_{2}$, and $\theta_{3}$ are then calculated using inverse kinematics of the 3 DOF position $\Lambda_{3 p}^{-1}$ to align the hand tip position to ${ }^{0} p_{\mathrm{S} 7}[k+1]$. In Process 1 , 
$\theta_{4}, \theta_{5}$, and $\theta_{6}$ are fixed values. Therefore, inverse kinematics of 3 DOF are calculated considering that ${ }^{3} p_{S 7}$ is one link. Details are provided as shown below.

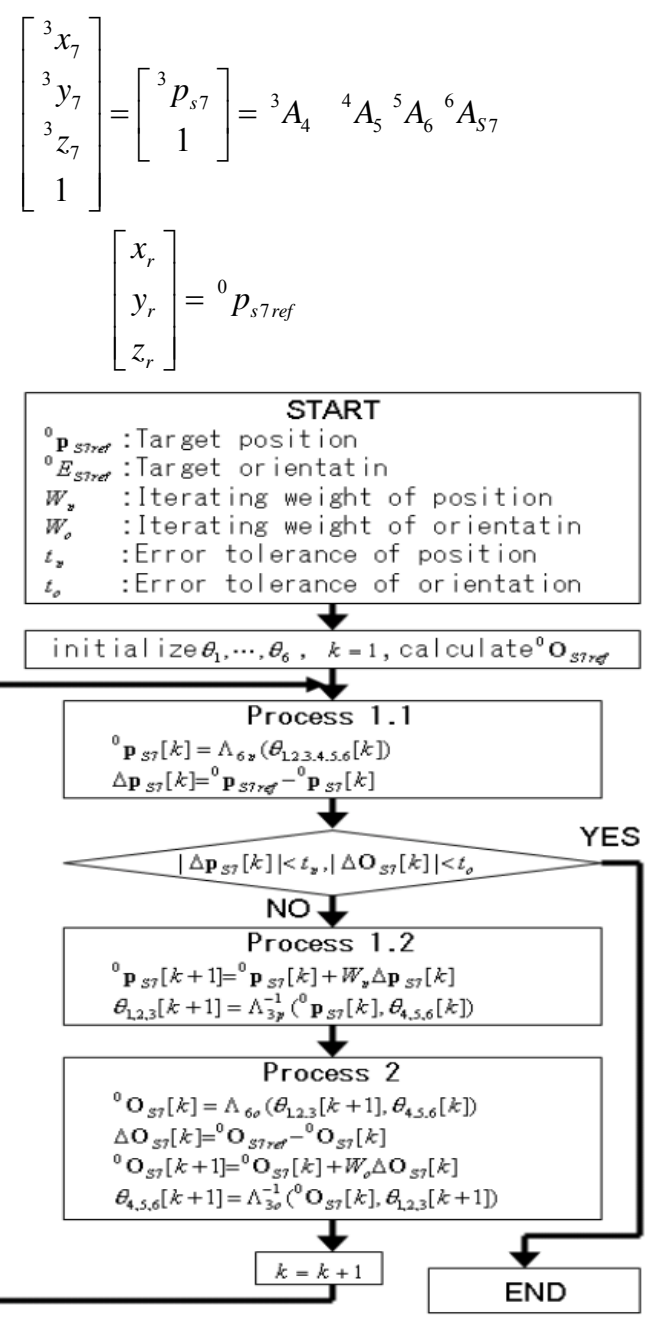

Fig.3 Flow chart of the Method of Sequential Retrieval

then four solutions presented in Table 2 are obtained using the following calculations. Although $\theta_{1}$ should be calculated prior to eq.(10) calculation, $y_{r 2}^{2}$ takes the same value even if either of $\theta_{1}$ is used. Therefore, any one might be selected and substituted.

$$
\begin{gathered}
L_{x_{r} y_{r}}=\sqrt{x_{r}^{2}+y_{r}^{2}} \\
\theta_{x_{r} / y_{r}}=\arctan 2\left(y_{r}, x_{r}\right) \\
\alpha=\sin ^{-1}\left(\frac{{ }^{3} x_{7}}{L_{x_{r} y_{r}}}\right)-\frac{\pi}{2} \\
L_{3 z_{7} 3_{y_{7}}}=\sqrt{{ }^{3} z_{7}{ }^{2}+{ }^{3} y_{7}{ }^{2}} \\
\theta_{3_{37} / 3_{y_{7}}}=\arctan 2\left({ }^{3} z_{7}+{ }^{3} y_{7}\right) \\
Z_{r 2}=z_{r}-L_{1} \\
y_{r 2}^{2}=\left(-x_{r} \sin \theta_{1}+y_{r} \cos \theta_{1}\right)^{2}
\end{gathered}
$$

$$
\begin{gathered}
\beta=\sin ^{-1}\left(\frac{y_{r 2}^{2}+z_{r 2}^{2}-L_{3_{z 3}}^{2} y_{y}-L_{2}^{2}}{2 L_{3_{z 7} y_{y}} L_{2}}\right)-\frac{\pi}{2} \\
L_{f e}=\sqrt{y_{r 2}^{2}+z_{r 2}^{2}} \\
\theta_{e / f}=\arctan 2\left(-L_{3_{z 7} 3_{y 7}} \sin \beta, L_{3_{z 7} 3_{y 7}} \cos \beta+L_{2}\right) \\
\gamma=\sin ^{-1}\left(\frac{z_{r 2}}{L_{f e}}\right)-\frac{\pi}{2} \\
\arctan 2(y, x)=\left\{\begin{array}{ccc}
\tan ^{-1}(y / x) & \text { if } & x>0 \\
\operatorname{sign}^{2}(y) \pi / 2 & \text { if } & x=0 \\
\tan ^{-1}(y / x)+\pi & \text { if } & x<0
\end{array}\right.
\end{gathered}
$$

\section{B. Convergence judgment}

In the convergence judgment, it is considered that convergence occurred when $\left|\Delta p_{s 7}\right|<t_{p}$ and $\left|\Delta O_{s 7}\right|<t_{0}$ are established, and that $\theta_{1}[k], \ldots, \theta_{6}[k]$ at that point of time are considered to be solutions.

\section{Classification of solutions}

Four solutions (Table 2) exist for inverse kinematics of the 3 DOF position, with two solutions shown in Table 3 for inverse kinematics of the 3 DOF posture. However, $\Lambda_{3 p}^{-1}$ and $\Lambda_{30}^{-1}$ of Fig.3 use only one solution. Therefore, only one solution is obtained through one-time convergence.

If the number of each solution is represented as $N_{3 p}(\in\{1,2,3,4\}), \quad N_{30}(\in\{1,2\})$, then there are eight combinations of $N_{3 p}$ and $N_{30}$; solutions of eight types will be obtained if all are subjected to the convergence calculation. In this paper, number $N_{\text {sol }}$ of the solution of the RPY-type inverse kinematics is expressed as $N_{\text {sol }}=2\left(N_{3 o}-1\right)+N_{3 p}$, and numbers 8 are assigned to each solution.

\section{Application of numerical values (Example obtaining eight solutions)}

It is said that with an efficient solution, eight or fewer solutions are obtained for one target value. This can be verified using concrete numerical values if eight solutions are obtained, even if modification weights are used.

The length of each link is shown in Table 1.

TABLE 1 LINK LENGTH

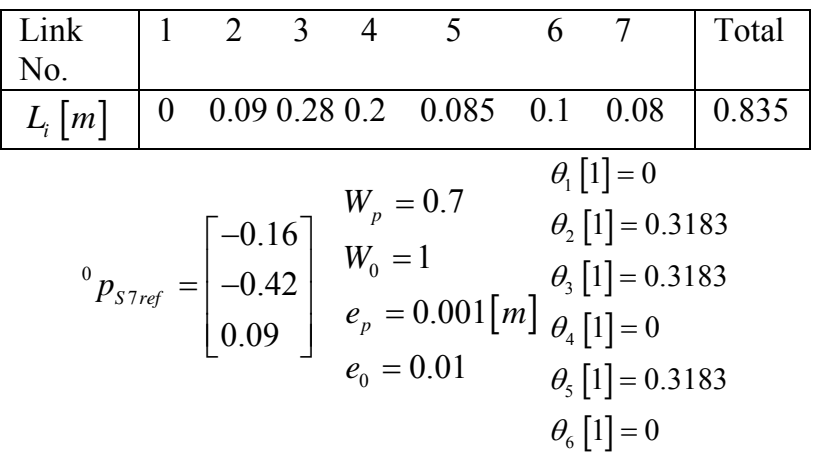




$$
{ }^{0} O_{\text {S7ZY Zref }}=(257,178,262)[\mathrm{deg}]
$$

The hand tip target positio ${ }^{0} P_{S 7 r e f}$,modification weight $W_{p}, W_{o}$, allowable error $e_{p}, e_{o}$, initial joint angle $\theta_{1}[1], \ldots \ldots, \theta_{6}[1]$, target posture ${ }^{0} O_{\text {S7ZY Zref }}$ are set as follows:

The allowable error of the position is $0.12 \%$ as compared with the whole arm length $0.835[\mathrm{~m}]$. The initial joint angle is set to such a value avoiding peculiar posture in accordance with the Newton-Raphson method because a peculiar posture is taken when all angles are $0[\mathrm{rad}]$ and because calculation of Jacobian determinant by Newton-Raphson method is not possible, as explained later.

Using the conditions described above, the solution of inverse kinematics is obtained using the Method of Sequential
Retrieval. Consequently, solutions of eight types are obtained as shown in Table 2

TABle2 Angle values $([\mathrm{rad}])$ of Obtained Solutions

\begin{tabular}{|l|c|c|c|c|c|c|}
\hline NO. & $\theta_{1}$ & $\theta_{2}$ & $\theta_{3}$ & $\theta_{4}$ & $\theta_{5}$ & $\theta_{6}$ \\
\hline 1 & 2.7335 & 5.1566 & 4.9733 & 1.1615 & 2.9943 & 4.2970 \\
\hline 2 & 2.7883 & 4.1525 & 1.3347 & 1.7191 & 2.7877 & 2.5952 \\
\hline 3 & 5.9293 & 2.1287 & 4.9450 & 4.8632 & 2.7881 & 2.5894 \\
\hline 4 & 5.8757 & 1.1270 & 1.3064 & 4.3039 & 2.9934 & 4.2941 \\
\hline 5 & 2.6429 & 5.8709 & 4.3503 & 4.2479 & 0.2419 & 1.0979 \\
\hline 6 & 2.5690 & 3.4925 & 1.9039 & 4.9814 & 0.5326 & 5.7971 \\
\hline 7 & 5.7109 & 2.7906 & 4.3789 & 1.8398 & 0.5322 & 5.7968 \\
\hline 8 & 5.7835 & 0.4098 & 4.9328 & 1.1061 & 0.2437 & 1.0959 \\
\hline
\end{tabular}

TABLE 3 TARGET VALUES AND RESULTS.

\begin{tabular}{|c|c|c|c|c|c|c|c|c|c|c|}
\hline $\mathrm{N}$ & \multicolumn{3}{|c|}{${ }^{0} p_{\text {S } 7 r e f}[m]$} & \multicolumn{3}{|c|}{${ }^{0} \mathrm{O}_{\text {S7ZY Zref }}[\mathrm{deg}]$} & \multicolumn{4}{|c|}{$W_{p}$} \\
\hline U & $\mathrm{X}$ & $\mathrm{y}$ & Z & Z & $\mathrm{Y}$ & Z & 1 & 0.7 & 0.4 & \\
\hline 1 & 0.11 & 0.10 & 0.31 & 180 & 288 & 67 & $\times$ & 0 & 0 & 0 \\
\hline 2 & -0.09 & 0.40 & 0.10 & 330 & 67 & 197 & $\times$ & $\times$ & 0 & 0 \\
\hline 3 & -0.02 & 0.03 & 0.32 & 332 & 287 & 47 & 0 & $\times$ & $\times$ & 0 \\
\hline 4 & -0.07 & -0.06 & 0.30 & 47 & 258 & 74 & $x$ & $\times$ & $\times$ & $x$ \\
\hline
\end{tabular}

\section{CONClusions}

To obtain solutions of inverse kinematics of an RPY-type robot arm, the Method of Sequential Retrieval is proposed in this paper. The following results were obtained.

1) It was confirmed that a maximum of eight solutions are obtained using the Method of Sequential Retrieval.

2) Such an example demonstrates that a solution is not obtained with $W_{p}$ at a certain value, but a solution is obtained using another value.

3) By varying the $W_{p}$ values randomly for every repetition, solutions are obtainable for more target values.

\section{REFERENCES}

[1] D. Manocha, J. F. Canny. Efficient inverse kinematics for general 6R manipulators. IEEE Transactions on Robotics and Automation, vol. 10, no. 5, pp. 648-657, 1994

[2] J. Q. Gan, E. Oyama, E. M. Rosales, H. Hu. A complete analytical solution to the inverse kinematics of the Pioneer2 robotic arm. Journal of Robotica, vol. 23, no. 1, pp. 123-129, 2005.

[3] J. Guo, V. Cherkassky. A solution to the inverse kinemat- ics problem in robotics using neural network processing. In proceedings of IEEE Conference on Neural Networks, Wash-ington, D.C., USA, pp. 299-304, 1989.

[4] S. Tejomurtula, S. Kak. Inverse kinematics in robotics using neural networks. Information Sciences, vol. 116, pp. 147-164, 1999.
[5] E. Oyama, A. Agah, K. F. MacDprman, S. Tachi. A modu-lar neural network architecture for inverse kinematics model learning. Neuro-computing, vol. 38-40, pp. 797-805, 2001.

[6] E. Papadopoulos, J. Poulakakis. Planning and model-based control for mobile manipulators. In proceedings of IEEE/RSJ International Conference on Intelligent Robots and Systems, Takamatsu, Japan, 1810-1815, 2000.

[7] D. Xu. A study on visual measurement and control for robot.Post-Doctoral Fellow Report, chapter 2, Institute of Automation, China, 2002.

[8] E. M. Rosales, J. Q. Gan. Forward and inverse kinematicsmodels for a 5-DOF pioneer 2 robot arm. Technical report,University of Essex, 2003.

[9] L. Zlajpah, B. Nemec. Kinematic control algorithms for on-line obstacle avoidance for redundant manipulators. IEEE/RSJ International Conference on Intelligent Robots and Systems, Lausanne, Switzerland, 1898-1903, 2002.

[10] M. A. Meggiolaro, G. Scriffignano, S. Dubowsky. Manipula-tor calibration using a single endpoint contact constraint. In Proceedings of ASME Design Engineering Technical 